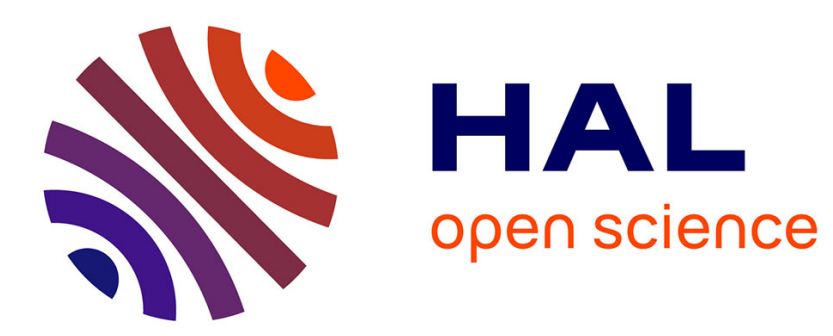

\title{
Universal ratios of critical amplitudes for the shear viscosity \\ P. Calmettes
}

\section{To cite this version:}

P. Calmettes. Universal ratios of critical amplitudes for the shear viscosity. Journal de Physique Lettres, 1979, 40 (20), pp.535-538. 10.1051/jphyslet:019790040020053500 . jpa-00231682

\section{HAL Id: jpa-00231682 https://hal.science/jpa-00231682}

Submitted on 1 Jan 1979

HAL is a multi-disciplinary open access archive for the deposit and dissemination of scientific research documents, whether they are published or not. The documents may come from teaching and research institutions in France or abroad, or from public or private research centers.
L'archive ouverte pluridisciplinaire HAL, est destinée au dépôt et à la diffusion de documents scientifiques de niveau recherche, publiés ou non, émanant des établissements d'enseignement et de recherche français ou étrangers, des laboratoires publics ou privés. 


\title{
Universal ratios of critical amplitudes for the shear viscosity
}

\author{
P. Calmettes \\ DPh-G/PSRM, CEN Saclay, B.P. n 2, 91190 Gif-sur-Yvette, France
}

(Reçu le 27 juin 1979, accepté le 4 septembre 1979)

Résumé. - L'analyse des mesures de viscosité existant déjà pour le mélange binaire, d'acide isobutyrique et d'eau donne des rapports d'amplitudes correspondant aux prévisions théoriques de Peliti.

\begin{abstract}
An analysis of the available shear viscosity data for the critical mixture of water and isobutyric acid gives amplitude ratios in agreement with the theoretical prediction of Peliti.
\end{abstract}

In recent years the shear viscosity of critical fluids has attracted an increasing amount of attention. Many different interpretations of the experimental data have been given, conflicting with the universality expected for the behaviour of critical systems.

A careful analysis [1] of previously published data has resolved this apparent difficulty. The shear viscosity $\eta$ of both pure and binary fluids near the critical point has been found to follow the scaling law

$$
\eta(n, t) \simeq \eta^{0}(n, t) t^{-y_{\eta}} f_{\eta}(x) \text { with } f_{\eta}(\infty) \equiv 1
$$

where $t=\frac{T-T_{\mathrm{c}}}{T_{\mathrm{c}}}$ and $n=\frac{N-N_{\mathrm{c}}}{N_{\mathrm{c}}}$, respectively, are the reduced temperature and the reduced order parameter. The scaling parameter is $x=t[B /|n|]^{1 / \beta}$ where $B$ and $\beta$ are the leading amplitude and the exponent, respectively, of the coexistence curve

$$
|n| \simeq B(-t)^{\beta} \text {. }
$$

The exponent $y_{\eta}$ and the function $f_{\eta}(x)$ have been found to be universal [1]. The best experimental estimate for the exponent is $y_{\eta}=0.0398 \pm 0.0005$, in agreement with theoretical expectations [2]. The amplitude $\eta^{0}(n, t)$ has been found to be a regular function of both $n$ and $t$, which reflects the behaviour of the viscosity in the absence of criticality [1].

It is worth noticing that, for binary mixtures, corrections to the leading behaviour (1) are not necessary to account for the experimental data, but, for pure fluids, nonanalytic corrections may be present. It has been shown [1, 3] that for ethane these corrections may be important. In any case, additive regular backgrounds are found to be smaller than the experi- mental uncertainties indicating that these contributions are very weak, if present at all. This result implies a weakness in the assumption that the background viscosity should be the viscosity in the absence of criticality [4].

Following this analysis, Peliti was able to show [5] that the scaling function $f_{\eta}(x)$ of the shear viscosity is simply related to the universal scaling function $f_{\chi}(x)$ of the order parameter susceptibility :

$$
\chi(n, t)=\Gamma(n, t) t^{-\gamma} f_{\chi}(x) \quad \text { with } \quad f_{\chi}(\infty) \equiv 1
$$

This relation is :

$$
f_{\eta}(x) \simeq\left[f_{\chi}(x)\right]^{y_{\eta} / \gamma} .
$$

According to mode-coupling theory this expression is exact but according to renormalization group theory the left-hand side is smaller than the righthand side. However, the difference is within $1 \%$ [5].

As we have already shown [3] expression (3) is in good agreement with the experimental function $f_{\eta}(x)$ given in figure 2 of ref. [1] for $x>0$. We found that $f_{\eta}(x)$ is about $3.5 \%$ lower than $\left[f_{\chi}(x)\right]^{y_{\eta} / \gamma}$ for small values of $x$, but this difference is not significant owing both to experimental errors and to the theoretical approximations which have been made. A more careful analysis of all the data available for the 3methylpentane-nitroethane mixture leads to a deviation of about $1 \%$, in rather good agreement with the theoretical result (3). Nevertheless, it is worth noticing that such an analysis can be affected by small contributions from possible non-analytic corrections and by errors in the parameters $\mathbf{B}$ and $\beta$ describing the coexistence curve. 
Another way to test the above prediction is to evaluate the critical viscosity amplitudes along the critical isochore, the critical isotherm and the coexistence curve. According to expression (3) two of the ratios of these amplitudes are expected to be universal constants at the critical point.

The shear viscosity has the following behaviour :

$$
\eta(0, t)=\eta_{+}^{0}(t) t^{-y_{n}}
$$

along the critical isochore $(x=\infty)$,

$$
\eta(n, 0)=\eta_{\sim}^{0}(n)\left[\frac{|n|}{B}\right]^{-y_{\eta} / \beta}
$$

along the critical isotherm $(x=0)$, and

$\eta\left[n \simeq B(-t)^{\beta}\right]=\eta_{-}^{0}(n, t)^{-y_{\eta}}=\eta_{-}^{0}(n, t)\left[\frac{|n|}{B} \mid\right]^{-y_{\eta} / \beta}$

along the coexistence curve $(x=-1)$.

Then, according to expression (3)

and

$$
\frac{\eta_{-}^{0}(0,0)}{\eta_{+}^{0}(0,0)}=\left[\frac{\Gamma_{-}(0,0)}{\Gamma_{+}(0,0)}\right]^{y_{\eta} / \gamma}
$$

$$
\frac{\eta_{\sim}^{0}(0,0)}{\eta_{-}^{0}(0,0)}=\left[\frac{\Gamma_{\sim}(0,0)}{\Gamma_{-}(0,0)}\right]^{y_{\eta} / \gamma}
$$

where $\Gamma_{+}(n, t), \Gamma_{\sim}(n, t)$ and $\Gamma_{-}(n, t)$, respectively, are the leading amplitudes of the susceptibility along the three previous paths.

These viscosity amplitude ratios are universal. Their theoretical values are given in table I. They have been inferred from the values of the susceptibility amplitude ratios reported by Tarko et al. [6] for a high temperature series calculation and by Brezin et al. [7] for a renormalization group calculation using, respectively, the values of the static exponents due to Zinn-Justin [8] and to Le Guillou et al. [9]. Finally, the experimental value $y_{\eta}=0.040 \pm 0.001$ has been used for the shear viscosity exponent. Typical uncertainties are $\pm 0.2 \%$ for the series values of table $\mathrm{I}$.

Contrary to the analysis of ref. [3], a knowledge of the parameters $B$ and $\beta$ describing the coexistence boundary is not required to experimentally evaluate the two amplitude ratios (4). Furthermore, when

Table I. - Theoretical and experimental values for

\begin{tabular}{|c|c|c|c|}
\hline Amplitude & Theoret & al values & Experimental \\
\hline ratios & H.T. series $\left({ }^{a}\right)$ & $\varepsilon$-expansion $\left({ }^{b}\right)$ & values \\
\hline- & - & - & - \\
\hline$\eta_{-}^{0} / \eta_{+}^{0}$ & 0.950 & 0.949 & $0.947 \pm 0.012$ \\
\hline$\eta_{\sim}^{0} / \eta_{-}^{0}$ & 0.986 & 0.985 & $0.986 \pm 0.003$ \\
\hline
\end{tabular}
the shear viscosity amplitude ratios of a critical fluid. Experimental values correspond to the mixture of isobutyric acid and water (data from ref. [10]). evaluating these ratios at the critical point, the small contributions from the possible non-analytic corrections are ruled out.

To our knowledge the only viscosity data which enable one to verify the theoretical results of table I are those of Woermann and Sarholz [10]. They have studied the mixture of water and isobutyric acid and have published numerical data both above and below $T_{\mathrm{c}}$. Results are given along isochores, i.e. for different concentrations. Linear extrapolation has been used to obtain viscosity values along the critical isotherm and the coexistence curve.

A plot of $\eta_{ \pm}^{0}(n, t)=\eta(n, t) t^{+y_{\eta}}$ versus $t$ is shown in figure 1 . Above and near $T_{\mathrm{c}}$ the viscosity amplitude varies linearly with the temperature

$$
\eta_{+}^{0}(0, t)=\eta_{+}^{0}(0,0)\left[1-a t+0\left(t^{2}\right)\right]
$$

with $a=7.5 \pm 0.4$. The critical amplitude is

$$
\eta_{+}^{0}(0,0)=1.974 \pm 0.005 \mathrm{cPo} .
$$

The uncertainties correspond to one standard deviation for the four most distant points from $T_{\mathrm{c}}$ shown in figure 1. For the three most distant points from $T_{\mathrm{c}}$ the peak-to-peak uncertainties are about ten times smaller. Notice that, for the purpose of calculating amplitude ratios, the absolute accuracy of the measurements is irrelevant.

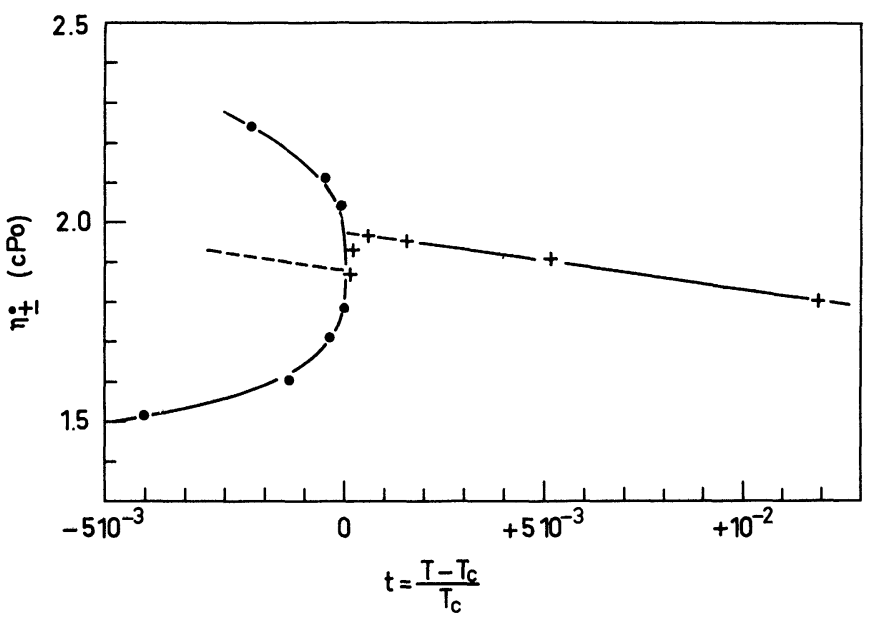

Fig. 1. - The shear viscosity amplitudes $\eta_{ \pm}^{0}(n, t)$ versus the reduced temperature $t$ for the critical mixture of water and isobutyric acid. (Data from ref. [10] : + above $T_{\mathrm{c}}, \bullet$ below $T_{\mathrm{c}}$ )

The two nearest points to the critical point have been neglected. Indeed, owing to experimental uncertainties, these data are too close to the critical point to ensure that they actually correspond to the critical isochore. As shown in figure 1 the nearest data point to $T_{\mathrm{c}}$ seems to be associated with the coexistence curve. Furthermore they can be affected by shear [11].

Below $T_{\mathrm{c}}$ the amplitude $\eta_{-}^{0}(n, t)$ has two different values along the two branches of the coexistence curve. 
The diameter amplitude

$$
\eta_{\mathrm{D}}^{0}(n, t)=\frac{\eta_{-}^{0}(n, t)+\eta_{-}^{0}(-n, t)}{2}
$$

is used to obtain the critical value $\eta_{-}(0,0)$ which is found to be

$$
\eta_{-}^{0}(0,0)=1.87 \pm 0.02 \mathrm{cPo} .
$$

To obtain this value a more simple and precise method is to plot the quantity $\eta_{-}^{0}(n, t)=\eta(n, t) t^{+y_{n}}$ versus $n$ and to linearly interpolate for the critical concentration $(n \equiv 0)$. The above value for the uncertainty on $\eta_{-}^{0}(0,0)$ has been obtained in this way. It is a peak-to-peak value.

The ratio of the two critical amplitudes $\eta_{+}^{0}(0,0)$ and $\eta_{-}^{0}(0,0)$ is given in table I. It agrees well with the theoretical values.

As shown in figure 1 the diameter amplitude $\eta_{\mathrm{D}}^{0}(n, t)$ has nearly the same temperature dependence as $\eta_{+}^{0}(0, t)$. Therefore below $T_{\mathrm{c}}$ the viscosity amplitude can be written as follows

$$
\eta_{-}^{0}(n, t)=\eta_{-}^{0}(n, 0)\left[1-a t+0\left(t^{2}\right)\right]
$$

with $a \simeq 7.5$.

A plot of

$$
\frac{\eta(n, 0)}{\eta\left[n=B(-t)^{\beta}\right]}=\frac{\eta_{\sim}^{0}(n, 0)}{\eta_{-}^{0}(n, 0)[1-a t]}
$$

versus $n$ is shown in figure 2 . Notice that to within $\pm 0.3 \%$ the data points lie on a curve similar to the coexistence curve. This behaviour is to be ascribed

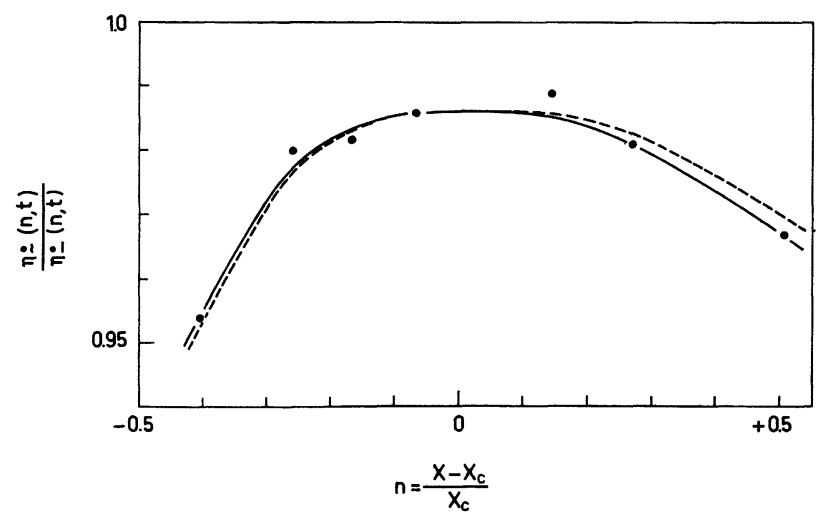

Fig. 2. - The shear viscosity amplitude ratio $\eta_{ \pm}^{0}(n, t) / \eta_{-}^{0}(n, t)$ versus the reduced mole fraction $n$ (see text). The points represent the experimental values inferred from the data of ref. [10]. The full line is an interpolation through the data points. The broken line is an hand-drawn curve similar to the coexistence curve. to the term $-a t=a(|n| / B)^{1 / \beta}$ in the denominator of the above expression. Furthermore, this similarity implies that, along the critical isotherm and the coexistence curve, the viscosity amplitudes have the same concentration dependence to within $\pm 0.3 \%$.

Using these features to interpolate the above amplitude ratio at critical concentration we obtain

$$
\frac{\eta_{\sim}^{0}(0,0)}{\eta_{-}^{0}(0,0)}=0.986 \pm 0.003
$$

which is in very good agreement with the theoretical values. It should be stressed that this ratio is directly inferred from the experimental data without any assumptions. Consequently the error in its experimental value is likely to be smaller as shown in figure 2 .

It is worth noticing that the scaling function conjectured in ref. [4]

$$
f_{\eta}(x)=\left[f_{\xi}(x)\right]^{y_{\eta} / v}
$$

where $\xi(n, t)=\xi^{0}(n, t) t^{-v} f_{\xi}(x)$ is the correlation length and is different from expression (3). Expression (5) is a crude exponentiation [12] of the result given by simple mode-coupling theory [13]. Using the universal constants of ref. [6-9] and expression (5) the following viscosity amplitude ratios are obtained

$$
\frac{\eta_{-}^{0}}{\eta_{+}^{0}}=\left\{\begin{array}{l}
0.959 \\
0.961
\end{array} \text { and } \frac{\eta_{\sim}^{0}}{\eta_{-}^{0}}=\left\{\begin{array}{l}
0.996 \\
0.994
\end{array}\right.\right.
$$

the upper values corresponding to a high temperature series calculation and the lower to a renormalization group calculation. For the upper values the uncertainties still amount to about $0.2 \%$. These two sets of conjectured values show that expression (5) does not correctly represents the data. This is particularly clear for the ratio $\eta_{\sim}^{0} / \eta_{-}^{0}$.

In conclusion we find that the theoretical prediction (3) due to Peliti is well founded. More precisely, using experimental data for a critical binary mixture we have shown that the shear viscosity amplitude ratios are nearly equal to the theoretical values of the osmotic compressibility amplitude ratios raised to the power $y_{\eta} / \gamma \simeq 0.032$. Since it has already been shown that the scaling law (1) represents the experimental viscosity data [3] quite well for any value of the scaling parameter, the behaviour of the shear viscosity of critical fluids can be now regarded as well understood.

Acknowledgements. - We acknowledge stimulating discussions with Luca Peliti. 


\section{References}

[1] C.almettes, P., Phys. Rev. Lett. 39 (1977) 1151

[2] Siggia, E. D., Halperin, B. I. and Hohenberg, P. C., Phys. Rev. B 13 (1976) 2110 ;

Ohta, T. and Kawasaki, K., Prog. Theor. Phys. 55 (1976) 1384 ;

Garisto, F. and Kapral, R., Phys. Rev. A 14 (1976) 884.

[3] CAlmettes, P., Thesis, University of Paris (June 1978) ; CEA, S.R.M. Report no 1545 .

C.Almettes, P., International Conference on Critical Dynamics, Geneva, Swizerland, April 1979.

[4] Basu, R. S. and Sengers, J. V., J. Heat Trans. 101 (1979) 3.

[5] Peliti, L., Private communication (1977) and to appear in...
[6] Tarko, H. B. and Fisher, M. E., Phys. Rev. B 11 (1975) 1217

[7] Brezin, E., Le Guillou, J. C. and Zinn-Justin, J., Phys. Lett. 47A (1974) 285.

[8] Zinn-Justin, J., Submitted to J. Physique.

[9] Le Guillou, J. C. and Zinn-Justin, J., Phys. Rev. Lett. 39 (1977) 95.

[10] Woermann, D. and Sarholz, W., Ber. Bunsenges. Phys. Chem. 69 (1965) 319.

[11] Oxтовy, D. W., J. Chem. Phys. 62 (1975) 1463.

[12] Ohta, T., J. Phys. C 10 (1977) 791.

[13] D'Arrigo, G., Mistura, L. and Tartaglia, P., J. Chem. Phys. 66 (1977) 80. 